Dongqing Wang • Bingquan Wu • Yan Li • Wanjie Heng

Haohao Zhong • Ying Mu • Jiajin Wang

\title{
A Chinese homozygote of familial hypercholesterolemia: identification of a novel C263R mutation in the LDL receptor gene
}

Received: October 30, 2000 / Accepted: December 18, 2000

\begin{abstract}
Familial hypercholesterolemia (FH) is an autosomal dominant disorder caused by mutations in the low-density lipoprotein receptor $(L D L R)$ gene; it is characterized by a high concentration of LDL, which frequently gives rise to tendon xanthomas and premature coronary artery disease (CAD). Individuals with heterozygous $\mathrm{FH}$ in China often exhibit a milder phenotype than those in other countries. The diagnosis of heterozygous $\mathrm{FH}$ relies on the clinical phenotype and this does not always permit unequivocal diagnosis of the disease. In the course of investigation of $\mathrm{FH}$ in a Chinese population sample, we found a family whose proband showed a markedly raised concentration of LDL cholesterol in plasma, and the presence of skin and tendon xanthomata. We used single-strand conformation polymorphism (SSCP) analysis to screen all the 18 exons and the exon-intron boundaries of the $L D L R$ gene. One novel homozygous mutation, replacing $\mathrm{T}$ by $\mathrm{C}$ at nucleotide 850 in exon 6 was identified. This change substituted cysteine for arginine at codon 263 (C263R) of the LDLR. By means of mutant allele-specific amplification, we unequivocally diagnosed six heterozygotes with this novel mutation in the proband's family.
\end{abstract}

Key words Familial hypercholesterolemia LDL receptor · Lipoproteins $\cdot$ Mutations $\cdot$ Phenotype

\section{Introduction}

Mutations in the gene for the low-density lipoprotein (LDL) receptor cause familial hypercholesterolemia (FH), an autosomal dominant disorder. Patients with typical

D. Wang $(\triangle) \cdot$ B. Wu $\cdot$ Y. Li $\cdot$ W. Heng $\cdot$ H. Zhong

Department of Pathology, Medical Department of Peking

University, 38 Xueyuan Road, Beijing, P.R. China

Tel. +86-010-62092407; Fax +86-010-62091406

e-mail: dqw2000@263.net

$\mathrm{Y}$. Mu $\cdot$ J. Wang

Department of Heredity, The People's Hospital, Peking University,

Beijing, P.R. China forms of heterozygous $\mathrm{FH}$ are characterized by high serum LDL cholesterol levels, which are usually associated with premature coronary heart disease (CHD) and tendon xanthomas in early middle age, while homozygous FH individuals frequently suffer fatal CHD by their third decade (Goldstein et al. 1995). To date, more than 200 different mutations of the $L D L R$ gene have been characterized worldwide (Hobbs et al. 1990; Hobbs et al. 1992; Varret et al. 1997). Chinese individuals with heterozygous FH are often recognized by virtue of their being parents of offspring with mutations in both alleles of the $L D L R$ gene (Mak et al. 1998). Unlike their heterozygous parents, the Chinese homozygous FH patients are as severely affected, as are those elsewhere (Sun et al. 1994). In this article, we present an investigation of a Chinese family whose proband, with severe hypercholesterolemia, carried a novel mutation of the $L D L R$ gene in a homozygous form, while the other six patients in the family, who carried this mutation in a heterozygous form, have a milder phenotype of FH. The main objective of our analysis was to delineate the full spectrum of mutations that underline $\mathrm{FH}$ in the Chinese population; our work represents a first step towards the implementation of nationwide DNA testing for this disease.

\section{Subjects and methods}

Subjects and lipoprotein measurement

The proband, a 3-year-old boy, was clinically diagnosed with homozygous FH based on a markedly increased concentration of plasma LDL cholesterol $(16.20 \mathrm{mmol} / \mathrm{l})$, and the presence of skin and tendon xanthomas. None of the other family members had a medical history of coronary artery disease (CAD) and tendon xanthomas. Venous blood samples were collected from the family members after an overnight fast of 12 to $14 \mathrm{~h}$. Total plasma cholesterol, triglycerides, and high-density lipoprotein cholesterol (HDL-C) (determined after precipitation of apolipoprotein B-containing lipoproteins with heparin $/ \mathrm{MnCl}_{2}$ ) were 
measured by standard enzymatic techniques (Sveger et al. 1987). LDL-C concentration was calculated by the Friedewald formula (Friedewald et al. 1972).

Single-strand conformation polymorphism (SSCP) and sequencing analysis

Genomic DNA for polymerase chain reaction (PCR) amplification was extracted from whole blood. Exons 1-18 of the $L D L R$ gene were amplified by PCR, using oligonucleotide primers homologous to the intron sequences flanking the exons. When a variant band was revealed by SSCP analysis, the PCR products were purified, and sequence analysis of the corresponding DNA fragment was performed, using an Applied Biosystems Model 377 automated DNA sequencer (Perkin-Elmer Cetus, Foster, CA, USA) on both strands to identify the nature of the mutation. Further confirmation of the mutation was achieved on the basis of mutant allele-specific amplification (MASA) analysis.

Statistical analysis

Plasma lipoprotein levels in mutation carriers and noncarriers were compared using non-parametric (Student-
Newman-Keuls) analysis. Values are given as means \pm SDs of the mean. Statistical significance was considered to be at $P$ values of less than 0.05 .

\section{Results and discussion}

The DNA sample from the proband was screened by SSCP for mutation in all 18 exons of the $L D L R$ gene, and the aberrant migration pattern of the PCR product of exon 6 identified by DNA sequencing indicated that this subject was homozygous for a T-to-C substitution at nucleotide 850 (Fig. 1); this change is predicted to result in a substitution of cysteine for arginine at codon 263. C263R was the only sequence variation detected in the entire coding with splicesite consensus sequences of the $L D L R$ gene of the proband.

Diagnosis of the C263R mutation was carried out by mutation allele-specific amplification in all 16 members of the proband's family from whom genomic DNA was available. This assay demonstrated the presence of the mutation in 6 other members of the family in heterozygous form, while the other 9 individuals were excluded from carrier status. The pedigree structure of C263R, and the ages and untreated lipoprotein profiles of the 16 family members examined, are shown in Fig. 2. The mean $( \pm S D)$ serum

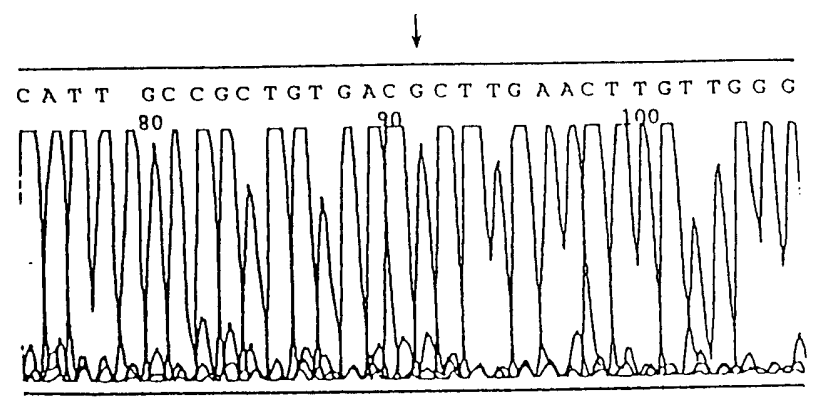

proband

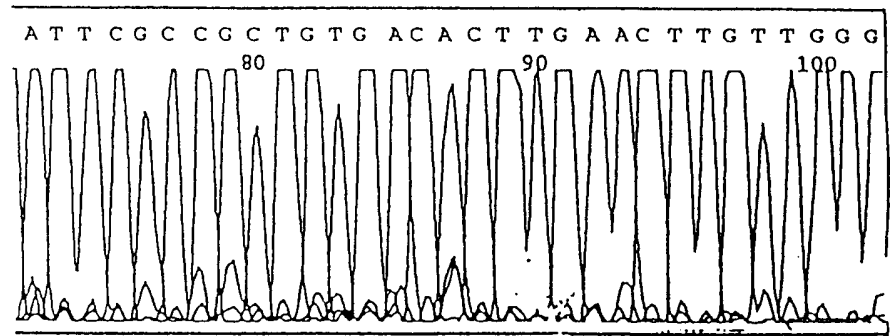

normal

Fig. 1. sequencing results of the C263R homozygous mutation and results for a normal control. Arrow indicates the identified nucleotides

Fig. 2. Partial pedigree and mutation analysis of the proband's family. Arrow indicates the proband. Square symbols indicate males; circles, females. The half-filled symbols indicate heterozygosity for the C263R mutation in exon 6. TC, Total cholesterol; $T G$, plasma triglyceride; $H D L-C$, high-density lipoprotein cholesterol; $L D L-C$, low-density lipoprotein cholesterol; Xan, tendon xanthomata; $C A D$, coronary artery disease; $I D$, number

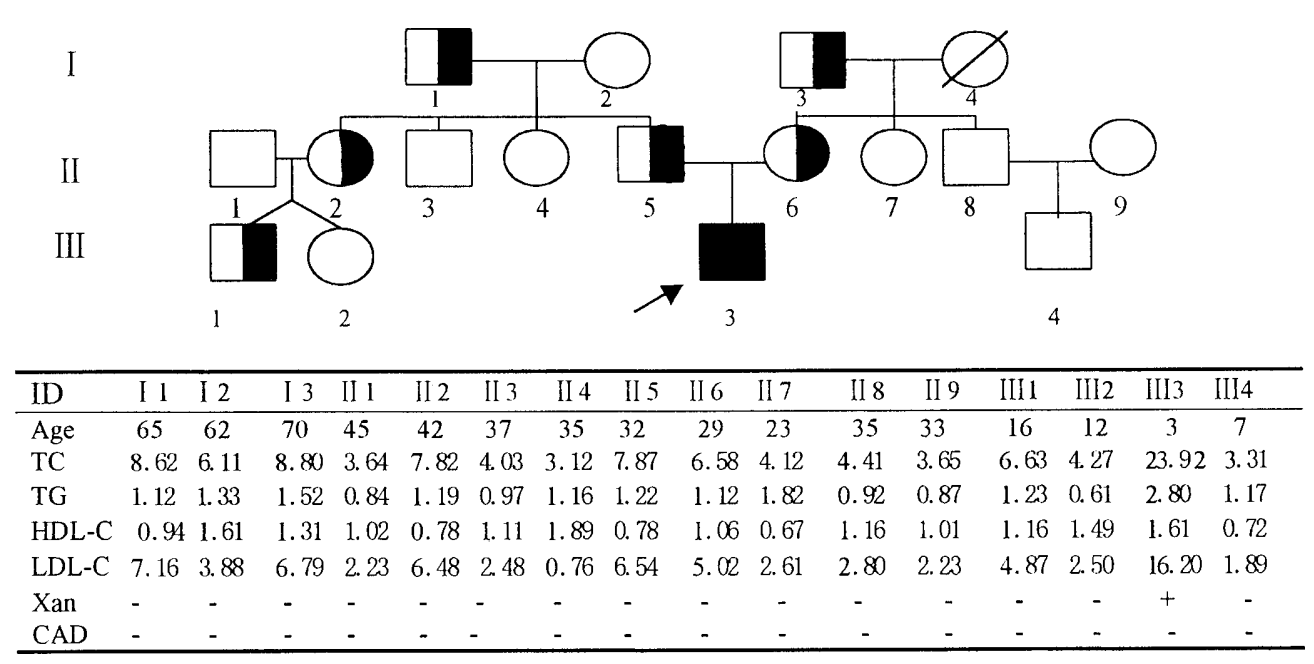


LDL-C levels in heterozygous mutation carriers $(n=6)$ and non-carriers $(n=9)$ in the family were $6.14 \pm 0.96$ and 2.37 $\pm 0.82 \mathrm{mmol} / 1$, respectively. The difference was significant $(P<0.05)$. Screening for mutation was conducted in 100 apparently healthy control subjects randomly selected from the Beijing Red Cross Blood Center. These subjects were examined to exclude the possibility that the missense mutation C263R is a polymorphism in the Chinese population.

The Cys $\rightarrow$ Arg substitution at codon 263 described in this article represents a novel mutation in exon 6 of the $L D L R$ gene. Exon 6 encodes part of the ligand-binding domain (LBD) of the LDL receptor protein (Yamamoto et al. 1984). The 292-amino acid LBD of the receptor, encoded by exons 2-6, is a cysteine-rich sequence composed of seven tandem repeats, each containing 40 amino acids. Each of the seven repeats contains six evenly spaced cysteine residues which are involved in intra-repeat disulfide bonding. The cosegregation of the C263R mutation and the FH phenotype is not unequivocal. To address the question of whether or not the C263R is an FH-causing mutation, several lines of evidence strongly suggest that the Cys $\rightarrow$ Arg substitution causes hypercholesterolemia in this family. First, the substitution in this region is expected to interfere with the normal intra-repeat disulfide bonding in this region of the protein, thereby destabilizing the protein structure. Secondly, loss of cysteine residues in the LBD has been reported to consistently produce deleterious effects on protein function. The third, perhaps most compelling line of evidence implicating the Cys $\rightarrow$ Arg substitution as the cause of FH in this family, is the finding that the substitution segregated only with clinically affected family members, and was not detected in clinically unaffected family members. Furthermore, the mutation was not observed in 100 random human DNA samples, indicating that it is not a common polymorphism in the Chinese population. To further characterize $\mathrm{C} 263 \mathrm{R}$ expression of the mutation, investigation of the LDLR protein in vitro and linkage analysis in extended pedigrees are needed.

In this study, we first found a novel homozygous mutation (C263R) of the LDLR in a 3-year-old boy from Henan province with a clinical diagnosis of homozygous FH. However, his parents and other affected individuals in the kin- dred do not exhibit evidence of tendon or skin xanthomata, and apparently do not have any increased risk of CAD. Based on their clinical presentation, the heterozygous patients in this family may be diagnosed as 'possible FH'. We speculate that the absence of a characteristic phenotype of heterozygous FH was, most likely, a result of factors other than the primary mutation, at least in this family. Therefore, there is a need for accurate and distinct criteria for diagnosing heterozygous $\mathrm{FH}$ in China.

Acknowledgments We thank Professor Jiajin Wang at the Department of Heredity, The People's Hospital, Peking University, for providing patients' materials. This work was supported by the Science Foundation of the Ministry of Health, P.R. China.

\section{References}

Friedewald WT, Levy RI, Fredrickson DS (1972) Estimation of the concentration of low-density lipoprotein cholesterol in plasma without the use of the preparative ultracentrifuge. Clin Chem 18:499-502

Goldstein JL, Hobbs H, Brown MS (1995) Familial hypercholesterolemia. In: Scriver CR, Beaudet AL, Sly WS, Valle D (eds) The metabolic and molecular bases of inherited diseases. McGraw-Hill, New York, pp 1981-2030

Hobbs HH, Russell DW, Brown MS, Goldstein JL (1990) The LDL receptor locus in familial hypercholesterolemia: mutational analysis of a membrane protein. Annu Rev Genet 24:133-170

Hobbs HH, Brown MS, Goldstein JL (1992) Molecular genetics of the LDL receptor gene in familial hypercholesterolemia. Hum Mut 1:445-466

Mak YT, Pang CP, Tomlinson B, Zhang J, Chan YS, Mak TW, Masarei JR (1998) Mutations in the low-density lipoprotein receptor gene in Chinese familial hypercholesterolemia patients. Arterioscler Thromb Vasc Biol 18:1600-1605

Sun XM, Patel DD, Webb JC, Knight BL, Fan LM, Cai HJ, Soutar AK (1994) Familial hyperchlesterolemia in China. Identification of mutations in the LDL-receptor gene that result in a receptor-negative phenotype. Arterioscler Thromb 14:85-94

Sveger T, Fex G, Borgfors N (1987) Hyperlipidemia in school children with family history of premature coronary heart disease. Acta Paediatr 76:311-315

Varret M, Rabes JP, Collod-Beroud G, Junien C, Boileau C, Beroud C (1997) Software and database for the analysis of mutations in human LDL receptor gene. Nucleic Acids Res 25:172-180

Yamamoto T, Davis CG, Brown MS, Schneider WJ, Casey ML, Goldstein JL, Russell DW (1984) The human LDL receptor: a cysteine-rich protein with multiple Alu sequences in its mRNA. Cell 39:27-38 